Fetal Diagnosis

and Therapy
Fetal Diagn Ther 2018;43:19-25

DOI: $10.1159 / 000461580$
Received: August 23, 2016

Accepted after revision: February 8, 2017

Published online: March 11, 2017

\title{
Incidence and Causes of Intentional Fetal or Neonatal Demise in Twin-Twin Transfusion Syndrome
}

\author{
Marjolijn S. Spruijt $^{\mathrm{a}}$ Ellen Tameeris ${ }^{\mathrm{a}}$ De-Peng Zhao ${ }^{\mathrm{a}}$ Johanna M. Middeldorp ${ }^{\mathrm{b}}$ \\ Monique C. Haak ${ }^{\mathrm{b}}$ Dick Oepkes $^{\mathrm{b}}$ Enrico Lopriore ${ }^{\mathrm{a}}$ \\ ${ }^{a}$ Division of Neonatology, Department of Pediatrics, and b Division of Fetal Medicine, Department of Obstetrics, \\ Leiden University Medical Centre, Leiden, The Netherlands
}

\section{Keywords}

Twin-twin transfusion syndrome - Termination of pregnancy - Selective fetal reduction - Withdrawal of neonatal intensive care · Fetoscopic laser therapy · Cerebral injury

\begin{abstract}
Introduction: The aim of this study is to evaluate the incidence and causes of intentional fetal and neonatal demise in twin-twin transfusion syndrome (TTTS). Material and Methods: All TTTS pregnancies managed at our centre between 2000 and 2014 were included. We evaluated incidence and causes of intentional fetal/neonatal demise, defined as termination of pregnancy, selective fetal reduction, or withdrawal of neonatal intensive care. Results: Intentional fetal/neonatal demise occurred in $9.8 \%(110 / 1,122)$ of fetuses and was due to termination of pregnancy (2.2\%), selective fetal reduction (4.2\%), or withdrawal of neonatal intensive care (3.4\%). Reasons for termination of pregnancy included complications of laser treatment (72.0\%), severe fetal anomaly (20.0\%), and unwanted pregnancy (8.0\%). Reasons for selective fetal reduction were technical difficulties to perform laser surgery (51.1\%), fetal complications (38.3\%),
\end{abstract}

\section{KARGER}

E-Mail karger@karger.com

www.karger.com/fdt and parental preference for fetal reduction rather than laser treatment (10.6\%). Reasons for withdrawal of neonatal intensive care treatment were severe cerebral injury (47.4\%), severe pulmonary complications (15.8\%), birth asphyxia (5.3\%), multiple complications of TTTS and/or prematurity combined (21.1\%), or other (10.5\%). Conclusions: Intentional fetal or neonatal demise in TTS occurs frequently and is often due to complications after laser surgery and/or severe (cerebral) injury in affected fetuses or neonates.

\section{(C) 2017 The Author(s)}

Published by S. Karger AG, Basel

\section{Introduction}

Twin-twin transfusion syndrome (TTTS) results from imbalanced inter-twin blood flow through placental vascular anastomoses and is associated with an increased risk of fetal and neonatal mortality $[1,2]$. Treatment with fetoscopic laser surgery strongly reduces the risks of morbidity and mortality [3, 4]. Although short- and longterm outcome in survivors has improved over time owing to improvements in fetal and neonatal care, TTTS is still associated with an increased incidence of cerebral injury and neurodevelopmental impairment $[5,6]$.

Enrico Lopriore, $\mathrm{MD}, \mathrm{PhD}$

Division of Neonatology, Department of Pediatrics Leiden University Medical Centre, PO Box 9600

NL-2300 RC Leiden (The Netherlands)

E-Mail e.lopriore@lumc.nl 
The incidence of fetal or neonatal mortality as well as short- or long-term morbidity in TTTS after laser surgery varies greatly between reported cohorts $[4,7-12]$. These differences may be due to multiple reasons including methodological differences between the studies, variations in definitions of severe morbidity, type of laser treatment, and level of experience in fetal therapy centres. An additional reason could be related to varying rates of termination of pregnancy, selective fetal reduction, and withdrawal of neonatal care in cases with unfavourable prognosis and/or severe cerebral injury $[4,8,13]$. If severe fetal injury is detected during pregnancy, termination of pregnancy or selective feticide may be considered within the constriction of the law. If severe cerebral injury is detected close to or after delivery, withholding life-sustaining neonatal treatment or withdrawal of intensive care treatment may be taken into consideration. A higher rate of (intentional or unintentional) fetal or neonatal demise in cases with unfavourable prognosis will lead to a higher rate of mortality and indirectly to a lower rate of neurodevelopmental impairment in survivors due to selection. This important factor is usually not reported or discussed when reporting the long-term outcome in TTTS series. Decisions on intentional fetal or neonatal demise are influenced by cultural, legal, and social factors, and incidence rates are bound to be different between treatment centres in different countries.

The aim of this study is to describe the incidence and causes of termination of pregnancy, selective fetal reduction, and withdrawal of neonatal intensive care treatment in TTTS pregnancies treated at our centre during the past 15 years.

\section{Material and Methods}

We conducted a cohort study of all TTTS pregnancies treated at the Leiden University Medical Centre (LUMC) since the beginning of laser therapy at our centre in 2000. The LUMC is the single Dutch tertiary referral centre for TTTS.

All monochorionic twin pregnancies with TTTS referred to and treated at the LUMC between 2000 and 2014 were included in this study. Most TTTS pregnancies were treated with fetoscopic laser surgery. We also included TTTS pregnancies in which selective fetal reduction or termination of pregnancy was chosen as the primary management option. TTTS was diagnosed using standard prenatal ultrasound criteria and staged according to the criteria of Quintero [10,11, 14]. Triplets or higher-order multiple gestations were excluded from the study.

The following data were recorded for every individual fetus of the included pregnancies: year of TTTS treatment, Quintero stage, laser technique, donor or recipient, gender, fetal demise, mode of delivery, neonatal survival (up to 1 month after birth), and the presence of severe cerebral injury. Severe cerebral lesions were defined in the same way as in our previous study [15], summarized as the presence of at least one of the following: intraventricular haemorrhage grade III, periventricular haemorrhagic infarction, periventricular leukomalacia grade II or greater, porencephalic cysts, arterial stroke, ventricular dilatation, or a combination of these.

We recorded all cases with intentional demise during the fetal or neonatal period and reasons for these management choices. Intentional fetal demise included termination of pregnancy or selective feticide. Termination of pregnancy was defined as termination of both twins or of the surviving co-twin after spontaneous demise of the first. Selective fetal reduction was defined as termination of one fetus by means of bipolar umbilical cord coagulation, radiofrequency ablation, or the administration of intracardiac potassium chloride after laser therapy. Intentional neonatal demise was defined as the withholding of neonatal intensive care treatment (treatment is never started) or the withdrawal of life-sustaining treatment in severely ill neonates because of a suspected poor prognosis, resulting in death.

The primary outcome in this study was a composite outcome termed intentional demise. As a secondary outcome measure we analysed differences in the incidence of intentional demise over time, comparing three 5-year subcohorts: 2000-2004, 20052009 , and 2010-2014. Finally, we analysed the reasons for intentional fetal demise or neonatal demise and divided these into categories.

Importantly, a new national guideline concerning the care for extremely premature infants was implemented in the Netherlands in 2010 , advising active neonatal intervention for very preterm neonates born at gestational ages at or above $240 / 7$ weeks [16]. The previous national guideline advised active neonatal treatment only after $250 / 7$ weeks, unless there were compelling arguments to decide otherwise. This change in policy occurred therefore during the period of our study.

The primary composite outcome, intentional demise, was reported as a percentage of the total number of TTTS-affected fetuses and as a percentage of the total number of TTTS pregnancies. The secondary outcome was analysed by dividing the study cohort into three 5-year subcohorts. Analysis of the incidence of intentional demise over the studied years was calculated by $\chi^{2}$ test for trend. The final secondary outcome, reasons for intentional fetal and neonatal demise, was reported per category, i.e., termination of pregnancy, selective fetal reduction, and withdrawal of neonatal treatment, as a percentage of the total number of fetuses, pregnancies, and/or neonates per category. Statistical analysis was performed using SPSS Statistics version 23.0 (SPSS, Inc., Chicago, IL, USA).

No ethical approval was required for this anonymized retrospective study. The institutional review board of the LUMC reviewed the study protocol and declared a statement of no objection.

\section{Results}

A total of 561 twin pregnancies (1,122 fetuses) with TTTS treated at our centre with laser therapy or selective fetal reduction between 2000 and 2014 were included in
2

Fetal Diagn Ther 2018;43:19-25

DOI: $10.1159 / 000461580$
Spruijt/Tameeris/Zhao/Middeldorp/

Haak/Oepkes/Lopriore 
Table 1. Patient characteristics of the twin-twin transfusion syndrome cohort

\begin{tabular}{lc}
\hline Total number of pregnancies & 561 \\
Total number of fetuses & 1,122 \\
Gender & \\
Female & $542(48.3)$ \\
Male & $552(49.2)$ \\
Unknown & $28(2.5)$ \\
Delivery mode & \\
Caesarean section & $347(30.9)$ \\
Vaginal & $725(64.6)$ \\
Unknown & $50(4.5)$ \\
Gestational age at birth* & $33.0 \pm 3.5(24-42)$ \\
Birthweight* & $1,852 \pm 682(502-4,320)$ \\
Quintero stage (number of pregnancies) & \\
1 & $62(11.1)$ \\
2 & $190(33.9)$ \\
3 & $274(48.8)$ \\
4 & $26(4.6)$ \\
Unknown & $9(1.6)$ \\
Technique used at intervention (number of pregnancies) \\
Laser therapy ( $n=528$ pregnancies) \\
Sequential selective laser \\
Solomon technique & $311(58.9)$ \\
Selective fetal reduction $(n=47$ pregnancies) \\
Bipolar cord coagulation & $44(93.6)$ \\
Radiofrequency ablation & $1(2.1)$ \\
Intracardiac potassium (after laser) & $2(4.3)$ \\
\hline
\end{tabular}

Data are $n(\%)$ or mean \pm standard deviation (range). Denominator is total No. of fetuses unless otherwise specified. * Presented only for live-borns $>240 / 7$ weeks' gestation $(n=848)$.

this study. The baseline characteristics of these patients are shown in Table 1. In 29 cases, the outcome of the pregnancy or neonate(s) was unknown. In 147 cases, unintentional fetal demise occurred due to death of one or both twins after laser therapy or death of the co-twin after selective fetal reduction of the other fetus. In 50 cases, death occurred after spontaneous immature birth before gestational ages of $240 / 7$ weeks (see flowchart in Fig. 1).

Termination of pregnancy was performed after a mean gestational age of 20.0 weeks (range 16.0-23.4 weeks). The mean gestational age at which selective fetal reduction was performed was 19.9 weeks (range 13.9-33.0 weeks).

Selective fetal reduction was performed in the recipient in $68.1 \%(32 / 47)$ of cases and in the donor in the remaining $31.9 \%$ of cases. Neonatal intensive care was withdrawn in the donor in $60.5 \%(23 / 38)$ and in the recipient in the remaining $39.5 \%$ of neonates.

Incidence and Causes of Intentional Fetal or Neonatal Demise in TTTS

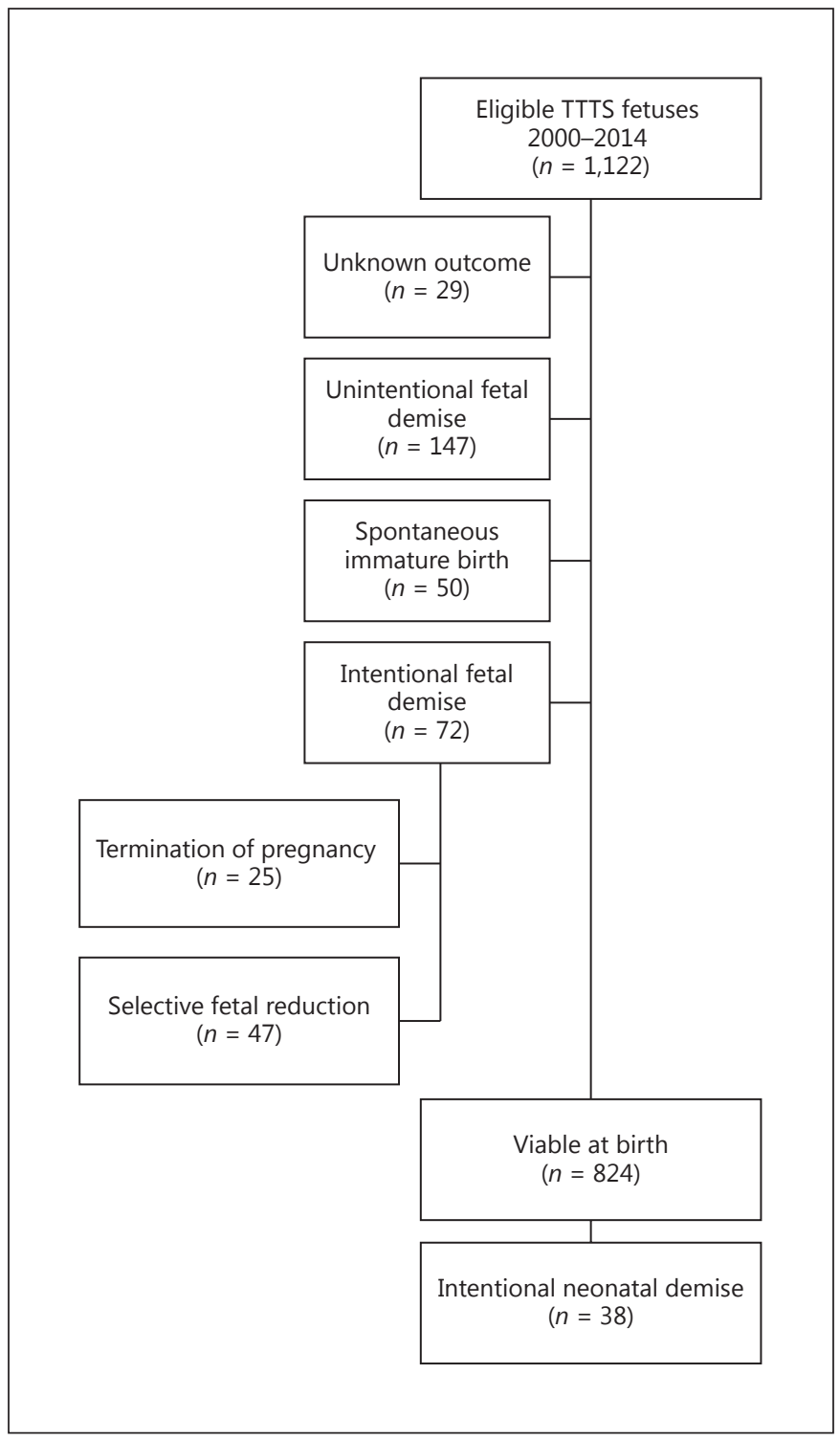

Fig. 1. Flow chart of study population. TTTS, twin-twin transfusion syndrome.

In 14 of 47 (29.8\%) pregnancies in which selective feticide was performed, prior laser therapy had taken place, but turned out to be incomplete (recurrent or persistent TTTS, reversal of TTTS, or twin anaemia-polycythaemia sequence occurred following laser therapy), after which re-intervention was necessary (not shown in table).

The technique used for selective fetal reduction was bipolar cord coagulation in 44 of 47 cases. Fetal intracardiac potassium chloride injection was used after laser surgery in two cases, and radiofrequency ablation was used in one fetus.

Fetal Diagn Ther 2018;43:19-25 DOI: $10.1159 / 000461580$ 
Table 2. Primary outcome: intentional fetal and neonatal demise

\begin{tabular}{lll}
\hline & $\begin{array}{l}\text { Fetuses } \\
(n=1,122)\end{array}$ & $\begin{array}{l}\text { Pregnancies } \\
(n=561)\end{array}$ \\
\hline $\begin{array}{lll}\text { Intentional fetal demise } \\
\quad \text { Selective fetal reduction }\end{array}$ & $72(6.4)$ & $62(11.1)$ \\
$\quad \begin{array}{l}\text { Termination of pregnancy } \\
\begin{array}{l}\text { Intentional neonatal demise } \\
\text { Total intentional fetal/neonatal }\end{array}\end{array}$ & $25(2.2)$ & $15(8.4)$ \\
$\quad$ demise & $110(9.8)$ & $32(5.7)$ \\
\hline
\end{tabular}

Data are presented as $n(\%)$.

\section{Primary Outcome}

The primary composite outcome, intentional fetal or neonatal demise, occurred in $9.8 \%(110 / 1,122)$ of fetuses (Table 2). When calculated per total number of pregnancies, intentional demise occurred in $16.8 \%$ (94/561) of pregnancies.

\section{Intentional Fetal Demise}

Intentional fetal demise occurred in $6.4 \%$ of fetuses $(72 / 1,122)$ and included selective fetal reduction in $4.2 \%$ $(47 / 1,122)$ and termination of pregnancy in $2.2 \%$ $(25 / 1,122)$ of fetuses. In five pregnancies, termination of pregnancy was performed after one fetus had already died unintentionally in utero.

\section{Intentional Neonatal Demise}

The incidence of intentional neonatal demise was $3.4 \%(38 / 1,122)$ of all fetuses, or $4.6 \%(38 / 824)$ of TTTS neonates who were alive at birth. In six pregnancies, withdrawal of neonatal care occurred in both co-twins; consequently, the number of unique pregnancies affected by intentional neonatal demise is reduced by six compared to the number of fetuses (or really, neonates) affected. One pregnancy was affected by selective fetal reduction of one co-twin and withdrawal of neonatal care in the other; therefore, the sum of the number of pregnancies affected by fetal and neonatal demise is reduced by one.

\section{Secondary Outcomes}

\section{Intentional Demise over Time}

The incidence of intentional fetal and neonatal demise did not change significantly over time. The incidence was
$8.8 \%$ (19 of 216 fetuses) in the first subcohort (2000$2004), 9.8 \%$ (41 of 420$)$ in the second (2005-2009), and $10.3 \%$ (50 of 486) in the third subcohort $(p=0.83)$.

\section{Reasons for Intentional Fetal and Neonatal Demise}

An overview of reasons for intentional demise is given in Table 3.

\section{Reasons for Termination of Pregnancy}

Severe complications after laser therapy were the most common reason to perform termination of pregnancy, as these were established in $72.0 \%$ (18/25) of involved fetuses. Complications included preterm prelabour rupture of membranes (PPROM) in 48.0\% (12/25). In all of these cases, PPROM was associated with severe oligohydramnios or anhydramnios, in some cases combined with imminent immature delivery. Evident intrauterine infection was the reason for termination of pregnancy in $20.0 \%$ $(5 / 25)$ of cases and these included two cases ( 4 fetuses) of severe maternal sepsis. Termination of pregnancy was performed after reversal of TTTS after laser surgery without the possibility of a successful second intervention in one pregnancy ( $4.0 \%$ of fetuses). In three pregnancies (5 living fetuses), termination of pregnancy was performed due to congenital or syndromal fetal abnormalities (not known at the time of laser treatment), i.e., trisomy 21 (1 pregnancy), Jeune syndrome, a rare skeletal dysplasia (1 pregnancy), and in one case bilateral clubfeet combined with cardiac hypertrophy, the latter most likely due to TTTS in a recipient (detected after unintentional fetal demise of the donor shortly after laser therapy). There was one unwanted pregnancy, which was terminated after the development of complications after laser therapy (severe growth retardation of one fetus, followed by twin anaemia-polycythaemia sequence after laser therapy).

\section{Reasons for Selective Fetal Reduction}

Selective feticide was performed in 47 TTTS pregnancies. In $51.1 \%$ (24/47), selective fetal reduction was chosen as the mode of therapy during the procedure (primarily planned as laser surgery) because complete laser surgery was impossible to achieve due to either anatomical or positional reasons (19 cases), or because of intra-amniotic bleeding occurring during the procedure, obscuring fetoscopic visualization (5 cases). Anatomical or positional causes included unfavourable (anterior) placental position, a stuck donor twin overlying the site of the vascular equator, and in one case, severe maternal obesity. A total of 528 pregnancies in our cohort were treated with laser therapy. These 24 cases treated with selective fetal
Fetal Diagn Ther 2018;43:19-25
DOI: $10.1159 / 000461580$
Spruijt/Tameeris/Zhao/Middeldorp/ Haak/Oepkes/Lopriore 
Table 3. Secondary outcome: reasons for intentional demise

\begin{tabular}{lcc}
\hline Termination of pregnancy & 25 fetuses & 15 pregnancies \\
Complications after laser therapy & $18(72.0)$ & $11(73.3)$ \\
Early PPROM & $12(48.0)$ & $7(46.7)$ \\
Intrauterine infection & $5(20.0)$ & $3(20.0)$ \\
$\quad$ Reversal of TTTS & $1(4.0)$ & $1(6.7)$ \\
Chromosomal or other severe anomaly of fetus(es) & $5(20.0)$ & $3(20.0)$ \\
Unwanted pregnancy and multiple complications after laser & $2(8.0)$ & $1(6.7)$ \\
\hline Selective fetal reduction & 47 fetuses & $19(40.4)$ \\
Complete laser therapy not possible (anatomical/positional) & $5(10.6)$ & \\
Complete laser therapy not possible (intra-amniotic bleeding) & $18(38.3)$ & $6(12.7)$ \\
Complications in one fetus & $6(12.7)$ & $1(2.1)$ \\
$\quad$ Severe cerebral injury & $5(10.6)$ & \\
Hydrops fetalis & $5(10.6)$ & \\
Multiple injury due to amniotic bands & 38 neonates \\
Congenital anomaly & $18(47.4)$ & \\
Choice of parents, no complications besides TTTS & $13(34.2)$ \\
\hline Withdrawal of neonatal intensive care treatment & $5(13.2)$ \\
Severe cerebral injury & $6(15.8)$ \\
$\quad$ Prenatally acquired & $8(21.1)$ \\
Postnatally acquired & $2(5.3)$ \\
Severe pulmonary complications & $1(2.6)$ \\
Multiple complications (of TTTS and prematurity, not cerebral) & $3(7.9)$ & \\
Hevere asphyxia & & \\
Deemed previable at birth, no active treatment & \\
\hline
\end{tabular}

Data are presented as $n(\%)$. PPROM, preterm prelabour rupture of membranes; TTTS, twin-twin transfusion syndrome.

reduction due to the impossibility to perform laser represent $4.3 \%(24 /(528+24))$ of pregnancies in which laser therapy was the intended treatment.

The reason for selective feticide was the occurrence of complications in one of the fetuses in $38.3 \%(18 / 47)$ of cases, including severe cerebral injury in 6 cases, hydrops fetalis in 6 cases, injury due to amniotic band syndrome in 1 fetus, and discordant congenital anomaly in 5 fetuses. The congenital anomalies seen in these five fetuses were double outlet right ventricle, severe lower urinary tract obstruction, bilateral multicystic kidney disease, gastroschisis, and anencephaly.

In $10.6 \%$ of cases (5/47), parents decided to perform selective fetal reduction as a primary treatment option to optimize the chance of having at least one healthy infant. These five pregnancies were staged as Quintero stages 1, 2 , and 3 and there were no additional complications besides TTTS nor any significant growth discordance in these fetuses.

Incidence and Causes of Intentional Fetal or Neonatal Demise in TTTS
Reasons for Withdrawal of Neonatal Care

Of the 38 cases in which intentional neonatal demise took place, the reason for cessation of neonatal intensive care was severe cerebral injury in $47.4 \%$ (18/38). In 13 of these 18 neonates, cerebral injury was acquired antenatally. One of these, a donor born at 26 0/7 weeks of gestation, had a grade 3 intraventricular haemorrhage at birth and in addition a hypoplastic right heart with severe pulmonary valve stenosis. The combination of severe prematurity, severe cerebral injury, and a complex congenital heart defect led to the decision to withdraw life-sustaining treatment.

Severe pulmonary complications were the reason to withdraw treatment in $15.8 \%(6 / 38)$ of neonates. These included 3 cases of pulmonary hypoplasia after PPROM following fetal therapy and 3 cases of severe pulmonary disease associated with prematurity. In eight neonates (21.1\%), the decision to stop life-sustaining treatment was made because of a combination of multiple complications of TTTS and/or prematurity (not including severe cerebral injury).

Fetal Diagn Ther 2018;43:19-25 DOI: $10.1159 / 000461580$ 
Severe birth asphyxia was the reason to abstain from further therapy in 2 cases. Both these neonates were former recipients after laser therapy, born after 34 and 38 weeks of gestation, respectively, and residual anastomoses were found in neither case.

One ex-donor was born with massive fetal hydrops at 31 2/7 weeks and resuscitation at birth was withheld. Three babies were born alive but did not receive intensive care treatment because they were deemed too immature due to national protocols concerning the management of extremely premature infants at that time. These included a pair of twins born after a gestational age of 24 2/7 weeks in 2005 and a baby born at 25 0/7 weeks in 2010. The cotwin of this last baby did receive treatment because he was vital at birth (Apgar scores of 6, 7, and 9), but treatment was withdrawn on the fifteenth day because of multiple complications including a candida sepsis.

\section{Discussion}

This study shows that intentional demise in TTTS occurs in $9.8 \%$ of fetuses or neonates and $16.7 \%$ of pregnancies. To our knowledge, there are no earlier reports on the incidences and causes of intentional demise in TTTS. These results are important as they may partly explain differences in mortality and morbidity rates between reported TTTS cohorts.

It is reasonable to assume that centres with a higher rate of intentional demise (mainly when due to unfavourable prognosis or cerebral injury) will have a higher rate of mortality but a lower rate of adverse neonatal and long-term neurodevelopmental outcome, because a selection takes place of fetuses with a better prognosis. Conversely, centres with a lower rate of intentional demise, possibly due to cultural or legal reasons, will most probably have a lower mortality rate, but a higher rate of adverse neonatal and longterm (neurological) complications in surviving neonates. Awareness on the impact of intentional demise of fetuses and neonates affected by TTTS is of crucial importance to gain insight into which factors contribute to neonatal and long-term neurodevelopmental outcome and in order to compare outcome data from different TTTS series.

Our study also shows that reasons for intentional demise were often related to a suspected unfavourable or poor prognosis in the majority of cases $[7,8,13]$. In about half of the pregnancies in which selective fetal reduction was carried out, laser coagulation was the planned treatment initially, but turned out to be impossible to perform during the procedure due to either fetal positions or im- paired visibility caused by intra-amniotic bleeding. These cases represent $4.3 \%$ of the total number of pregnancies in which laser was the intended treatment. Because leaving TTTS untreated exposes both fetuses at great risk for severe complications including death, selective feticide is usually chosen in these situations to give at least one twin a reasonable chance of disease-free survival.

In one TTTS pregnancy, the occurrence of complications, combined with the fact that the pregnancy was unwanted to begin with, led to the decision to terminate the pregnancy. In five TTTS pregnancies, parents opted for selective fetal reduction as a primary treatment, in order to optimize the chances of at least one healthy child, instead of laser coagulation of the vascular anastomoses. In these five pregnancies, there were no (severe) anomalies or growth restrictions in either of the co-twins at the time of treatment decision.

Reasons for withholding or withdrawing neonatal intensive care treatment were all related to unfavourable prognosis. In almost half of the 38 cases of intentional neonatal demise, the decision to stop treatment was made because of severe cerebral injury, of which the majority was acquired antenatally ( $72.2 \%$ of neonates with severe cerebral injury vs. $27.8 \%$ acquired postnatally), comparable to what we previously reported [15]. Termination of treatment in neonates with severe cerebral injury strongly affects the longterm neurodevelopmental outcome of a cohort of TTTS survivors. Other complications leading to withdrawal of neonatal intensive care were either directly related to TTTS or TTTS treatment (pulmonary hypoplasia after oligo- or anhydramnios, severe hydrops, extreme prematurity after PPROM), to severe prematurity, or to perinatal asphyxia.

In a recent study, we showed a significant improvement in survival and long-term neurodevelopmental outcome in TTTS cases treated at our centre during the past decade [17]. We hypothesized that improvement in long-term outcome may partly be due to increased awareness and detection of fetal or neonatal cerebral injury and associated increased intentional demise. However, as shown in this study, the rates of intentional fetal and neonatal demise in the three consecutive 5-year subcohorts between 2000 and 2014 were not different. Other factors, including improvement of obstetric and neonatal care for TTTS mothers and infants, could have contributed to the improvements in outcome.

In conclusion, intentional fetal and neonatal demise occurred in $9.8 \%$ of TTTS-affected fetuses in our centre. This high rate of intentional demise may result in a relatively favourable short-term and long-term neurodevelopmental outcome in survivors of TTTS.
6

Fetal Diagn Ther 2018;43:19-25

DOI: $10.1159 / 000461580$
Spruijt/Tameeris/Zhao/Middeldorp/

Haak/Oepkes/Lopriore 


\section{References}

1 Lutfi S, Allen VM, Fahey J, O'Connell CM, Vincer MJ: Twin-twin transfusion syndrome: a population-based study. Obstet Gynecol 2004;104:1289-1297.

2 Lewi L, Jani J, Blickstein I, Huber A, Gucciardo L, Van Mieghem T, Done E, Boes AS, Hecher K, Gratacos E, Lewi P, Deprest J: The outcome of monochorionic diamniotic twin gestations in the era of invasive fetal therapy: a prospective cohort study. Am J Obstet Gynecol 2008;199:514.e1-8.

3 Akkermans J, Peeters SH, Klumper FJ, Lopriore E, Middeldorp JM, Oepkes D: Twenty-five years of fetoscopic laser coagulation in twin-twin transfusion syndrome: a systematic review. Fetal Diagn Ther 2015;38: 241-253.

4 Huber A, Diehl W, Bregenzer T, Hackeloer BJ, Hecher K: Stage-related outcome in twintwin transfusion syndrome treated by fetoscopic laser coagulation. Obstet Gynecol 2006; 108:333-337.

5 Lopriore E, Oepkes D, Walther FJ: Neonatal morbidity in twin-twin transfusion syndrome. Early Hum Dev 2011;87:595-599.

6 van Klink JM, Koopman HM, van Zwet EW, Oepkes D, Walther FJ, Lopriore E: Cerebral injury and neurodevelopmental impairment after amnioreduction versus laser surgery in twin-twin transfusion syndrome: a systematic review and meta-analysis. Fetal Diagn Ther 2013;33:81-89.
7 Chmait RH, Kontopoulos EV, Korst LM, Llanes A, Petisco I, Quintero RA: Stage-based outcomes of 682 consecutive cases of twintwin transfusion syndrome treated with laser surgery: the USFetus experience. Am J Obstet Gynecol 2011;204:393.e1-6.

8 van Klink JM, Slaghekke F, Balestriero MA, Scelsa B, Introvini P, Rustico M, Faiola S, Rijken $M$, Koopman HM, Middeldorp JM, Oepkes D, Lopriore E: Neurodevelopmental outcome at 2 years in twin-twin transfusion syndrome survivors randomized for the Solomon trial. Am J Obstet Gynecol 2016;214:113. e1-7.

9 Rossi AC, Vanderbilt D, Chmait RH: Neurodevelopmental outcomes after laser therapy for twin-twin transfusion syndrome: a systematic review and meta-analysis. Obstet Gynecol 2011;118:1145-1150.

10 Senat MV, Deprest J, Boulvain M, Paupe A, Winer N, Ville Y: Endoscopic laser surgery versus serial amnioreduction for severe twinto-twin transfusion syndrome. N Engl J Med 2004;351:136-144.

11 Slaghekke F, Lopriore E, Lewi L, Middeldorp JM, van Zwet EW, Weingertner AS, Klumper FJ, DeKoninck P, Devlieger R, Kilby MD, Rustico MA, Deprest J, Favre R, Oepkes D: Fetoscopic laser coagulation of the vascular equator versus selective coagulation for twinto-twin transfusion syndrome: an open-label randomised controlled trial. Lancet 2014;383: 2144-2151.
12 Roberts D, Neilson JP, Kilby MD, Gates S: Interventions for the treatment of twin-twin transfusion syndrome. Cochrane Database Syst Rev 2014;1:CD002073.

13 Chmait RH, Kontopoulos EV, Jackson M, Horenstein J, Timor-Tritsch I, Quintero RA: Selective reduction using intravascular potassium chloride injection after laser surgery for twin-twin transfusion syndrome. Fetal Diagn Ther 2016;39:306-310.

14 Quintero RA, Morales WJ, Allen MH, Bornick PW, Johnson PK, Kruger M: Staging of twin-twin transfusion syndrome. J Perinatol 1999;19:550-555

15 Spruijt M, Steggerda S, Rath M, van Zwet E, Oepkes D, Walther F, Lopriore E: Cerebral injury in twin-twin transfusion syndrome treated with fetoscopic laser surgery. Obstet Gynecol 2012;120:15-20.

16 de Laat MW, Wiegerinck MM, Walther FJ, Boluyt N, Mol BW, van der Post JA, van Lith JM, Offringa M; Nederlandse Vereniging voor Kindergeneeskunde; Nederlandse Vereniging voor Obstetrie en Gynaecologie: Practice guideline "Perinatal management of extremely preterm delivery" (in Dutch). Ned Tijdschr Geneeskd 2010;154:A2701.

17 van Klink JM, Koopman HM, van Zwet EW, Middeldorp JM, Walther FJ, Oepkes D, Lopriore E: Improvement in neurodevelopmental outcome in survivors of twin-twin transfusion syndrome treated with laser surgery. Am J Obstet Gynecol 2014;210:540.e1-7.
Incidence and Causes of Intentional Fetal or Neonatal Demise in TTTS
Fetal Diagn Ther 2018;43:19-25

DOI: $10.1159 / 000461580$ 